\title{
Fibroblast growth factor 19 is correlated with an unfavorable prognosis and promotes progression by activating fibroblast growth factor receptor 4 in advanced-stage serous ovarian cancer
}

\author{
LINGLING $\mathrm{HU}^{1}$ and LANXIANG CONG ${ }^{1,2}$ \\ Departments of ${ }^{1}$ Gynecology and ${ }^{2}$ Reproductive Medicine, Linyi People's Hospital, \\ Linyi, Shandong 276000, P.R. China
}

Received May 20, 2015; Accepted July 22, 2015

DOI: $10.3892 /$ or.2015.4212

\begin{abstract}
Fibroblast growth factor receptor 4 (FGFR4) has been confirmed to be associated with the progression and prognosis of ovarian cancer, while the underlying mechanism has not been well elucidated and the clinical significance of its ligand, fibroblast growth factor 19 (FGF19), has not been explored. To study the clinical significance of FGF19 in advanced-stage serous ovarian cancer, we detected the expression of FGF19 and FGFR4 by immunohistochemistry (IHC), evaluated the correlation between FGF19 and clinicopathological factors by Chi-square $\left(\chi^{2}\right)$ test, and analyzed the association between FGF19, FGFR4 and the overall survival rate using the Kaplan-Meier method. As a result, we demonstrated that high expression of FGF19 and FGFR4 both predicted unfavorable prognosis $(\mathrm{P}=0.033$ and 0.018 , respectively), whereas FGF19-FGFR4 double high expression was a more sensitive prognostic factor of advanced-stage serous ovarian cancer $(\mathrm{P}<0.001)$. With experiments in vitro, we demonstrated that both recombinant FGF19 and secreted FGF19 promoted ovarian cancer proliferation and invasion by activating FGFR4 and the subsequent AKT-MAPK signaling pathway, suggesting that FGF19-FGFR4 signaling may autoactivate in a paracrine or autocrine manner. In conclusion, FGF19-FGFR4 double high expression was a more sensitive prognostic factor than FGF19 or FGFR4 alone in advancedstage serous ovarian cancer. The FGF19-FGFR4 signaling pathway can promote ovarian cancer proliferation and invasion by the AKT-MAPK signaling pathway, indicating that FGF19 could be a potential therapeutic drug target of advanced-stage serous ovarian cancer.
\end{abstract}

Correspondence to: Dr Lanxiang Cong, Department of Reproductive Medicine, Linyi People's Hospital, 27 Jiefang Road, Linyi, Shandong 276000, P.R. China

E-mail: bradforlinyi@163.com; conglanxiang2006@126.com

Key words: fibroblast growth factor 19, fibroblast growth factor receptor 4 , ovarian cancer, prognosis, proliferation, invasion

\section{Introduction}

Ovarian cancer is one of the most lethal malignancies among all gynecological tumors and results in the second highest number of cancer-related deaths among women worldwide (1). Ovarian cancer is a group of heterogeneous tumors, which comprise several histological subtypes: serous, mucinous, endometrioid, clear cell and undifferentiated carcinomas. High-grade serous carcinoma accounts for $\sim 80 \%$ of epithelial ovarian carcinomas and has the highest recurrence rate. Due to advances in surgical management and adjuvant chemotherapy, the survival rate of ovarian cancer patients has been markedly increased, but ovarian cancer still remains the most lethal cancer in gynecology with a 5-year overall survival of only 25-35\% and causes more than 15,000 deaths/year (2-4), which is the focus of ovarian cancer study for decades (5). The high mortality of ovarian cancer is partly due to clinical silence and high recurrence. Most patients are diagnosed at an advanced stage and the majority of patients, up to $75 \%$, eventually suffer recurrence, although they receive debulking surgery and standard chemotherapy (6). In conclusion, effective, predictive or prognostic biomarkers are urgently needed for early diagnosis and survival time prolongation.

In the human, fibroblast growth factors (FGFs) are heparin-binding proteins consisting of a family of 18 members, which are essential for signal transduction through interacting with cell surface-associated heparan sulfate proteoglycans $(7,8)$. FGFs are involved in many cellular processes such as proliferation, differentiation and angiogenesis, and their ectopic overexpression has been discovered in many types of cancers. FGFs function as signaling inducers by interacting with FGFRs. Fibroblast growth factor receptors (FGFRs) comprise five members, including four tyrosine kinase receptors (FGFR1, FGFR2, FGFR3 and FGFR4) and a non-tyrosine kinase receptor (FGFR5, also known as FGFRL1) (9). FGFR4 is demonstrated to bind with FGF1, FGF2, FGF4, FGF6, FGF8, FGF9, FGF16, FGF17, FGF18, FGF19, FGF21 and FGF23, but FGF19 and FGF21 have the highest affinity and specificity to FGFR4. Among the FGFR family, FGFR4 is distinguished in ovarian cancer because previous studies have determined that it is a prognostic biomarker and potential therapeutic target for ovarian cancer (10). However, the underlying mechanism 
of how FGFR4 leads to an unfavorable prognosis and how FGFR4 is stimulated and triggers the downstream signaling network has not been well elucidated.

In the present study, we hypothesized that FGFR4 functions in ovarian cancers as part of a signaling network in the cancer microenvironment and thus we detected the expression of FGFR4-specific ligand, FGF19, in advanced-stage serous ovarian cancer with immunohistochemistry (IHC). Furthermore, we evaluated the prognostic value of FGF19 with univariate and multivariate analyses. Using experiments in vitro, we demonstrated that FGF19 can be secreted and promotes ovarian cancer progression such as proliferation and invasion by activating FGFR4, indicating that the FGF19-FGFR4 paracrine pathway can be considered as a potential drug target in ovarian cancer therapy.

\section{Materials and methods}

Patients and follow-up. A total of 134 patients were diagnosed with advanced-stage serous ovarian cancer by routine pathology from 2002 to 2012 at Linyi Hospital and Yishui Central Hospital, which was the primary cohort. Advanced stage was identified as stage IIIB-IV in the International Federation of Gynecology and Obstetrics stage system according to a previous study (10). The validation cohort consisting of 74 samples was selected from the primary cohort with criteria as available tissue samples and follow-up of more than 3 months. Samples (tissues and blood samples) were obtained from the Pathology Department with prior consent of the patients or their families and by approval of the Institutional Clinical Ethics Review Board. The diagnoses of the validation cohort were confirmed by two senior pathologists.

Additionally, 15 pairs of fresh frozen tumor tissues and the corresponding adjacent non-tumor tissues were collected immediately after surgery with prior consent of the patients and preserved in liquid nitrogen. Real-time polymerase chain reaction (PCR) with primers of FGF19 and FGFR4 was used to detect the mRNA levels in the tumor and non-tumor tissues.

Cell culture and agents. Ovarian cancer cell lines SK-OV-3, HO8910, HO8910PM and OVCAR3, hepatocellular carcinoma cell line HepG2, breast cancer cell line MCF-7 and colorectal cell line SW480 were purchased from the Chinese Academy of Sciences Cell Bank (Shanghai, China). The cell line SK-OV-3 was cultured in McCoy's 5A medium (Sigma-Aldrich) and HO8910, HO8910PM and OVCAR3 cells were cultured in RPMI-1640 medium. HepG2, MCF-7 and SW480 were cultured in Dulbecco's modified Eagle's medium (DMEM), all of which were supplemented with $10 \%$ fetal bovine serum (FBS), $100 \mathrm{U} / \mathrm{ml}$ penicillin and $100 \mu \mathrm{g} / \mathrm{ml}$ streptomycin, and cultured under $5 \% \mathrm{CO}_{2}$ in a humidified incubator at $37^{\circ} \mathrm{C}$. Recombinant human FGF19 (rFGF19) protein was purchased from Sino Biological, Inc. (Beijing China). Antibodies used in our experiments were as follows: mouse monoclonal anti-FGF19 antibody was from R\&D Systems (Minneapolis, MN, USA), anti-FGFR4 (Phospho-Tyr642) antibody was from Signalway Antibody LLC. (College Park, MD, USA), anti- $\beta$-actin antibody was from Santa Cruz Biotechnology, Inc. (Santa Cruz, CA, USA). The remaining antibodies were all from Cell Signaling Technology, Inc. (Danvers, MA, USA).
IHC and evaluation. IHC was carried out by the streptavidin peroxidase complex method. Followed by deparaffinization with xylene and graded alcohol, endogenous peroxidase was inactivated by incubation in $3 \%$ hydrogen peroxide for $10 \mathrm{~min}$. After nonspecific blocking with $5 \%$ bovine serum albumin, the slides were incubated in the primary antibody at a dilution of 1:100 at $4^{\circ} \mathrm{C}$ overnight. After the primary antibody, samples were incubated with the corresponding secondary antibody at $37^{\circ} \mathrm{C}$ for $1-2 \mathrm{~h}$ and then in 3,3'-diaminobenzidine (DAB) solution until satisfactory staining was shown. Every slide was evaluated by two senior pathologists unaware of the clinical information. Cases without concurrence were re-evaluated by a third pathologist.

The score of the IHC staining was based on multiplication of the staining intensity and the positive cell percentage. The staining intensity was scored as negative (0), weak (1), moderate (2) and strong (3), and scores of the stained area were defined as follows: $0,<10 \% ; 1,10-30 \% ; 2,>30-50 \%$; and 3 , $>50 \%$ positive cells. The cut-off was arbitrarily defined as a score $\geq 4$ was considered as high FGF19 or FGFR4 expression; a score $<4$ was considered as low FGF19 or FGFR4 expression.

Western blotting. Expression of FGF19 and FGFR4 was detected by western blotting. Briefly, the cells were lysed in modified RIPA buffer (Tris $50 \mathrm{mM}, \mathrm{NaCl} 150 \mathrm{mM}$, Triton X-100 1\%, SDS $0.1 \%$, deoxycholate $0.5 \%$, sodium orthovanadate $2 \mathrm{mM}$, sodium pyrophosphate $1 \mathrm{mM}, \mathrm{NaF}$ $50 \mathrm{mM}$, EDTA $5 \mathrm{mM}$, PMSF $1 \mathrm{mM}$ and $1 \mathrm{X}$ protease inhibitor cocktail) on ice for $10 \mathrm{~min}$ for complete lysis, and then centrifuged at $10,000 \times \mathrm{g}$ for $30 \mathrm{~min}$. Protein concentration of the supernatant was measured by Bradford assay kit (Sangon Biotech Co., Ltd., Shanghai, China). An amount of $40 \mu \mathrm{g}$ of the extracted protein from each sample was used for electrophoresis in $10 \%$ sodium dodecyl sulfate-polyacrylamide gel electrophoresis (SDS-PAGE), and then transferred to an NC membrane (Pall Corporation). The membrane was incubated in $5 \%$ defatted milk for $1 \mathrm{~h}$, in diluted primary antibody overnight and in horseradish peroxidase-labeled secondary antibody for $2 \mathrm{~h}$ in order. Proteins were finally visualized using enhanced chemiluminescence western blotting detection reagents (Thermo Fisher Scientific, Waltham, MA, USA).

Quantitative real-time polymerase chain reaction ( $q R T-P C R$ ). RT-PCR was used to detect the mRNA levels of FGF19 and FGFR4. Primers were designed and purchased from Sigma-Aldrich as follows: FGF19 forward, 5'-GCACAGTTTG CTGGAGATCA-3' and reverse, 5'-ATCTCCTCCTCGAAA GCACA-3'; FGFR4 forward, 5'-AGCACCCTACTGGACAC ACC-3' and reverse, 5'-ACGCTCTCCATCACGAGACT-3'. Briefly, total RNA of ovarian cancer cells was first extracted from the cell lines with the RNeasy kit (Qiagen) and the concentration of total RNA was tested. Then qRT-PCR was achieved and relative gene expression was measured by the StepOnePlus Real-Time PCR system (Applied Biosystems) using the SYBR-Green method according to the manual. Glyceraldehyde 3-phosphate dehydrogenase (GAPDH) was applied as an internal control.

Small interfering RNA and transfection. Knockdown of FGF19 or FGFR4 was accomplished by small interfering 

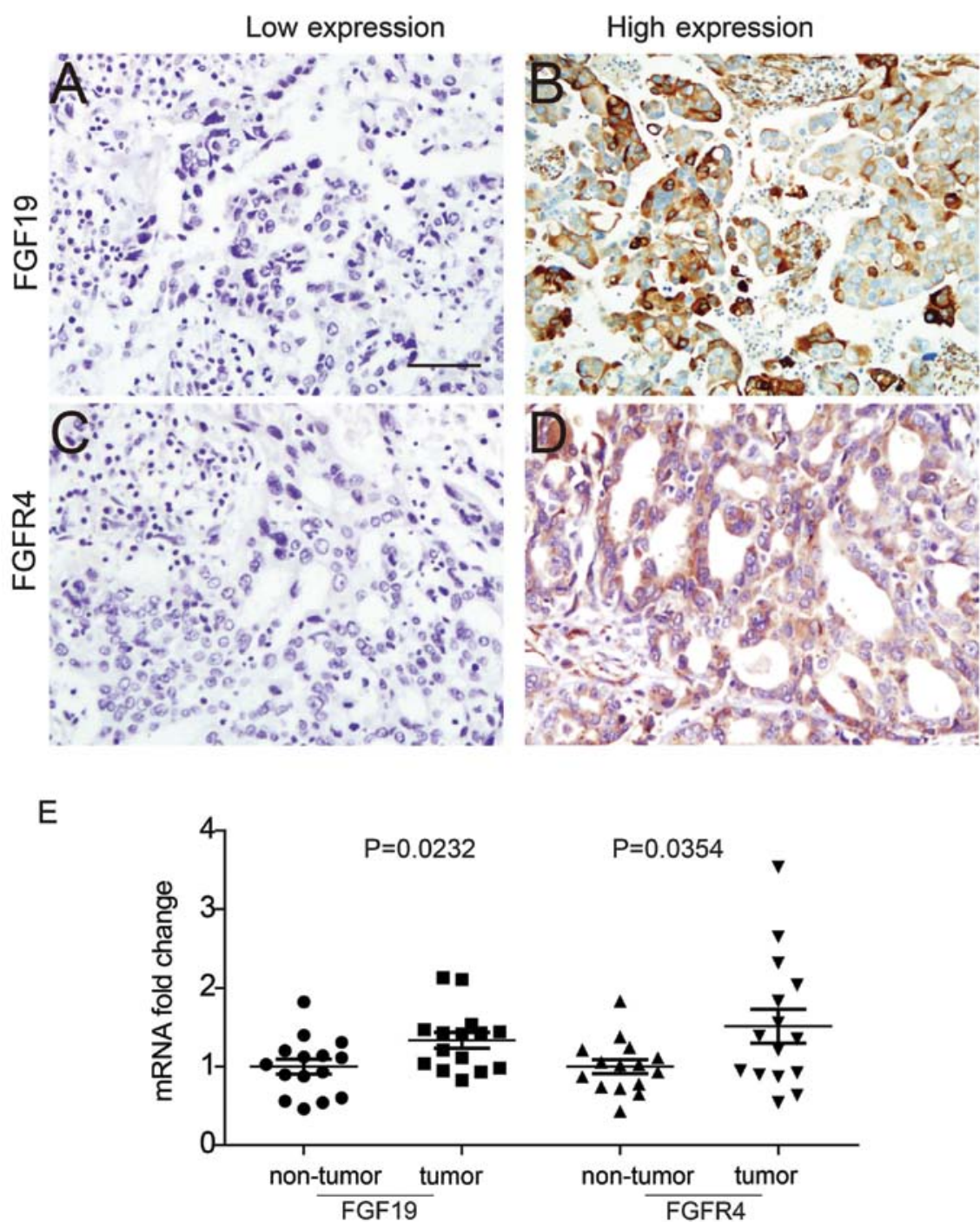

Figure 1. FGF19 and FGFR4 expression in serous ovarian cancer. (A) Low FGF19, (B) high FGF19, (C) low FGFR4 and (D) high FGFR4 expressions (scale bar, $50 \mu \mathrm{m}$ ). (E) Different mRNA levels of FGF19 and FGFR4 in tumor tissues and adjacent non-tumor tissues.

RNA (siRNA) purchased from Invitrogen (Carlsbad, CA, USA). The siRNA sequences of FGF19 and FGFR4 were obtained from Santa Cruz Biotechnology, Inc. Transfection of siRNA was realized with Lipofectamine 2000 (Invitrogen) according to the user guide. Results of the knockdown were validated by qRT-PCR and western blotting.

Cell proliferation assays. Cell proliferation was assessed by the MTT kit (Sangon Biotech Co., Ltd.). Briefly, an equal number of cells was planted into a 96-well plate and then starved for $6 \mathrm{~h}$ before stimulation. After stimulation with recombinant FGF19 or conditioned medium for the expected time, the proliferation was terminated by addition of $10 \mathrm{mg} / \mathrm{ml}$ MTT and then incubated at $37^{\circ} \mathrm{C}$ for $4-6 \mathrm{~h}$. After removing the superior carefully, the crystals at the bottom were resolved by $100 \mu 1$ DMSO and the absorbance was read at $490 \mathrm{~nm}$. The optical density (OD) at $490 \mathrm{~nm}$ was set as the baseline, and the proliferation index of the other groups was calculated by the OD490 ratio to the baseline. Every group had at least eight parallel wells, and analyzed data were from at least three independent experiments.
Matrigel invasion assays. Matrigel invasion assays were performed to evaluate the effect of FGF19 on cell invasion using $8-\mu \mathrm{m}$ pore size BD BioCoat Matrigel invasion chambers (Becton-Dickinson, Franklin Lakes, NJ, USA). After being planted into the upper chambers and starved for $6 \mathrm{~h}$, the cells were incubated in $10 \mathrm{ng} / \mathrm{ml}$ recombinant FGF19 protein of conditioned medium for $24 \mathrm{~h}$ and then fixed and stained with Diff-Quik stain. Non-invading cells in the upper chambers were wiped off while the cells in the lower chamber were counted using at least 8 random fields. The invaded number of cells without stimulation was counted and set as the baseline and the invasion index of the other groups was calculated as the ratio to the baseline. Analyzed data were from at least three independent experiments.

Enzyme-linkedimmunosorbent assay (ELISA) and conditioned medium. Serum FGF19 levels were measured with sandwich ELISA by the FGF19 Quantikine ELISA kit (R\&D Systems), following the manufacturer's instructions and previous studies $(11,12)$. FGF19 concentration in the cell medium was tested after treatment with $50 \mu \mathrm{M}$ chenodeoxycholic acid 
Table I. Characteristics of the patients with advanced-stage serous ovarian cancer.

\begin{tabular}{lcc}
\hline Characteristics & No. of patients & $\%$ \\
\hline Age (years) & & \\
$<50$ & 17 & 22.97 \\
$\geq 50$ & 57 & 77.03 \\
Lymph node metastasis & & \\
No & 37 & 50.00 \\
Yes & 37 & 50.00 \\
Histological grade & & \\
I & 11 & 14.86 \\
II & 24 & 32.43 \\
III & 39 & 52.70 \\
FGF19 & & \\
Low & 43 & 58.11 \\
High & 31 & 41.89 \\
FGFR4 & & \\
Low & 45 & 60.81 \\
High & 29 & 39.19 \\
FGF19+FGFR4 & & \\
Low & 58 & 78.38 \\
High & 16 & 21.62 \\
\hline
\end{tabular}

(CDCA) for $48 \mathrm{~h}$, and was then detected by an ELISA kit according to previous studies $(13,14)$. All samples from the cell culture media or serum were detected in duplicate.

To obtain efficient conditioned medium (CM), OVCAR3 cells were cultured in normal medium with $10 \%$ FBS for the expected time. Then $\mathrm{CM}$ was collected and filtered with a low protein-binding filter $(0.22 \mu \mathrm{m})$ (Millipore, Billerica, MA, USA). Amicon Ultra $15 \mathrm{ml}$ filters were used to concentrate the $\mathrm{CM}$ at 4,000 $\mathrm{g}$ when necessary. The same procedures were performed using SK-OV-3 cells as a control group. The CM was used immediately, stored at $4^{\circ} \mathrm{C}$ for a week or frozen at $-80^{\circ} \mathrm{C}$.

Statistical analysis. All data were analyzed by SPSS 17.0 software (SPSS, Inc., Chicago, IL, USA). Correlations between FGF19 and clinicopathological features were analyzed by $\chi^{2}$ test, and correlations between FGF19, FGFR4 and overall survival rates were calculated by the Kaplan-Meier method, and the difference between the high-expression and lowexpression group was analyzed by log-rank test. In experiments in vitro, differences between the control group and tested group were analyzed by the Student's unpaired t-test. $\mathrm{P}<0.05$ was considered to indicate a statistically significant result.

\section{Results}

Expression of FGF19 and FGFR4 in ovarian cancer. Expression of FGF19, the known specific ligand of FGFR4, was first detected in ovarian cancer samples with IHC. FGF19 expression was mainly observed in the cytoplasm, where it may
Table II. Correlation between FGF19 and clinicopathological features.

\begin{tabular}{lrrr}
\hline & \multicolumn{2}{c}{ FGF19 } & \\
\cline { 2 - 3 } Characteristics & Low & High & P-value \\
\hline Age (years) & 10 & 7 & 0.946 \\
$\quad<50$ & 33 & 24 & \\
$\geq 50$ & & & \\
Lymph node metastasis & 26 & 11 & 0.033 \\
No & 17 & 20 & \\
Yes & & & \\
Histological grade & 8 & 3 & 0.122 \\
I & 10 & 14 & \\
II & 25 & 14 & \\
III & & & \\
FGFR4 & 30 & 15 & 0.063 \\
Low & 13 & 16 & \\
High & & & \\
\hline${ }^{a}$ Chi-square test. & & & \\
\hline
\end{tabular}

be secreted out and function as a growth factor (Fig. 1A and B). FGFR4 expression was observed in both the membrane and cytoplasm (Fig. 1C and D). According to the criteria described in Materials and methods, expression of FGF19 and FGFR4 was divided into a high expression and low expression group. The percentage of samples with high-FGF19 and high-FGFR4 was 41.89 and $39.19 \%$, respectively (Table I). Moreover, to evaluate the significance of FGF19 and FGFR4 co-expression, we further defined FGF19-FGFR4 double high expression as cases having high expression for both FGF19 and FGFR4 expression, with the rest of the cases defined as the control group. The percentage of cases with FGF19-FGFR4 double high expression was $21.62 \%$ in our cohort.

Moreover, FGF19 and FGFR4 mRNA expression levels in tumor tissues and adjacent non-tumor tissues were detected and compared (Fig. 1E). The mRNA levels of FGF19 and FGFR4 in tumor tissues were demonstrated to be much higher than levels in the non-tumor tissues.

Clinical significance of FGF19. The correlation between FGF19 and clinicopathologic factors and the 5-year overall survival rates were analyzed to evaluate the clinical significance of FGF19 in ovarian cancer. Based on the $\chi^{2}$ test, we demonstrated that high expression of FGF19 was significantly associated with positive lymph node metastasis $(\mathrm{P}=0.033)$, indicating that FGF19 may play an important role in tumor invasion and metastasis. High FGF19 expression tended to be correlated with high FGFR4 expression, but the tendency was not statistically significant $(\mathrm{P}=0.063)$ (Table II).

Kaplan-Meier method was performed to further evaluate the prognostic value of detected clinicopathological parameters (Table III). High FGFR4 expression was demonstrated to be significantly associated with a poorer overall survival rate $(\mathrm{P}=0.018)$, which was consistent with a previous 
Table III. Correlation between clinicopathological features and the overall survival rate.

\begin{tabular}{|c|c|c|}
\hline Characteristics & 5-year survival rate $(\%)$ & P-value ${ }^{a}$ \\
\hline \multicolumn{3}{|l|}{ Age (years) } \\
\hline$<50$ & 39 & 0.967 \\
\hline$\geq 50$ & 31.7 & \\
\hline \multicolumn{3}{|c|}{ Lymph node metastasis } \\
\hline No & 54.3 & 0.002 \\
\hline Yes & 7.8 & \\
\hline \multicolumn{3}{|c|}{ Histological grade } \\
\hline $\mathrm{I}$ & 38.1 & 0.401 \\
\hline II & 37 & \\
\hline III & 31.4 & \\
\hline \multicolumn{3}{|l|}{ FGF19 } \\
\hline Low & 41.6 & 0.033 \\
\hline High & 20.6 & \\
\hline \multicolumn{3}{|l|}{ FGFR4 } \\
\hline Low & 41.8 & 0.018 \\
\hline High & 0 & \\
\hline \multicolumn{3}{|l|}{ FGF19+FGFR4 } \\
\hline Low & 37.9 & $<0.001$ \\
\hline High & 13.4 & \\
\hline
\end{tabular}

${ }^{\mathrm{a} L o g}$-rank test.

study (Fig. 2A) (10). In addition, we first proved that high FGF19 expression was closely correlated with unfavorable prognosis $(\mathrm{P}=0.033)$, suggesting that FGF19 could be considered as a prognostic biomarker and potential drug target (Fig. 2B). Notably, double high expression of FGF19 and FGFR4 was a better biomarker and predicted prognosis more reliably than FGF19 or FGFR4 alone (P<0.001) (Fig. 2C). FGF19-FGFR4 double high expression can predict poorer prognosis more accurately and sensitively than FGF19 or FGFR4 alone, indicating the promising and potential clinical significance of considering FGF19-FGFR4 double positivity as a biomarker. Moreover, the association between FGF19-FGFR4 and poor prognosis indicated that the FGF19-FGFR4 signaling pathway may promote cancer progression by a self-sufficient autocrine or paracrine pathway.

FGF19 and FGFR4 in ovarian cancer cells, medium and blood. Using experiments in vitro, we performed a series of functional assays to explore the mechanism explaining why high FGF19 and FGFR4 expression imply a much poorer prognosis. Expression levels of FGF19 and FGFR4 in ovarian cancers were first detected by western blotting. As shown in Fig. 3A, FGF19 and FGFR4 were widely expressed in ovarian cancer cell lines. SK-OV-3 had the lowest FGFR4 expression and HO8910 had the lowest FGF19 expression. OVCAR3 had relatively high FGF19 and FGFR4 expression, suggesting OVCAR 3 as a suitable model for a knockdown assay. To evaluate the role of FGF19 and FGFR4 in ovarian cancer progression, we regulated FGF19 and FGFR4 expression in OVCAR3 cells
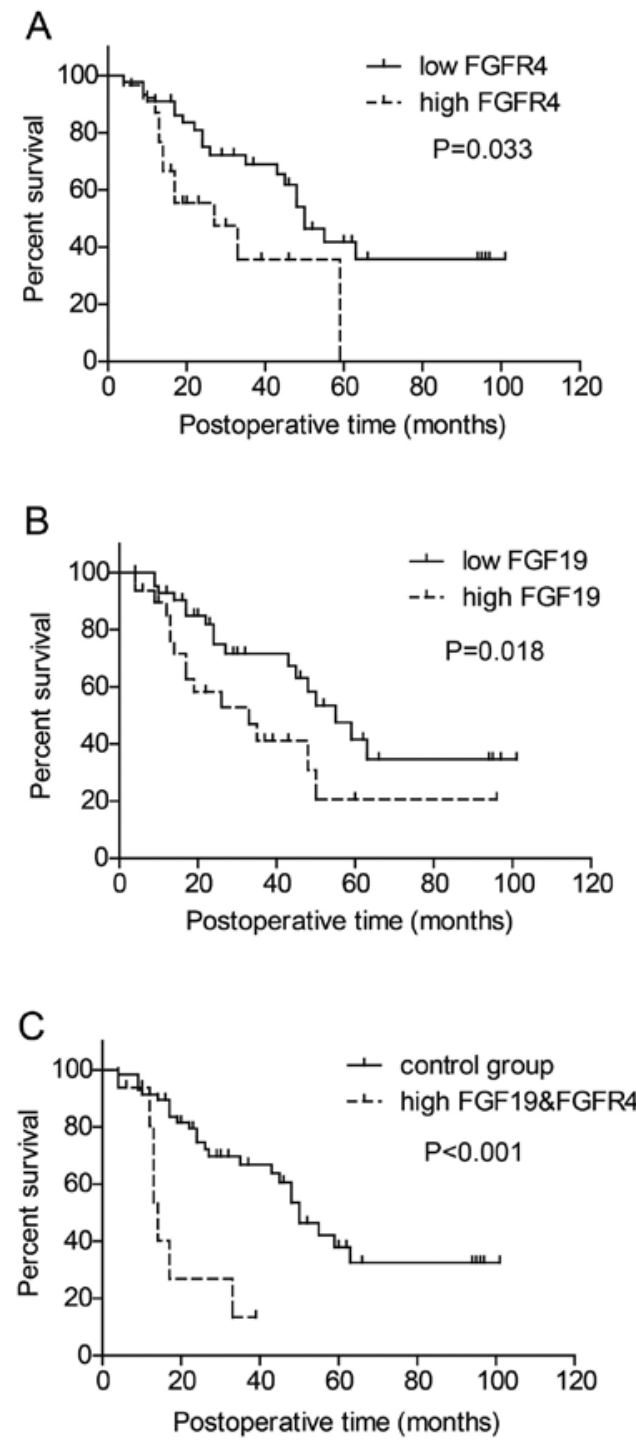

Figure 2. Correlation between the overall survival rate and FGFR4/FGF19. High (A) FGFR4 and (B) FGF19 expression can predict unfavorable prognosis of serous ovarian cancer. (C) FGFR4 and FGF19 double high expression is a more sensitive prognostic factor than FGFR4 and FGF19 alone.

by siRNA transfection and validated the successful knockdown of FGF19 (Fig. 3B and C) and FGFR4 (Fig. 3D and E) by qRT-PCR and western blotting. As a secreted growth factor, FGF19 was suspected to be secreted out from ovarian cancer cells. Thus medium of the SK-OV-3 and OVCAR3 cells, which had the lowest and highest FGF19 expression respectively, was used to detect the FGF19 concentration. After treatment with $50 \mu \mathrm{M}$ CDCA for $72 \mathrm{~h}, \mathrm{FGF} 19$ in both the SK-OV-3 and OVCAR3 medium was detectable (Fig. 3F). The FGF19 concentration in the OVCAR3 medium was markedly higher than that in the SK-OV-3 medium, which was consistent with the intracellular FGF19 content. After FGF19 was knocked down by siRNA, the concentration of FGF19 in the medium was significantly decreased compared to the control group, suggesting that its concentration in medium was directly related to the intracellular content. Based on the finding that the FGF19 is associated with prognosis and is secreted out from ovarian cancer, we hypothesized that FGF19 concentration in blood may predict earlier ovarian cancer progression and 


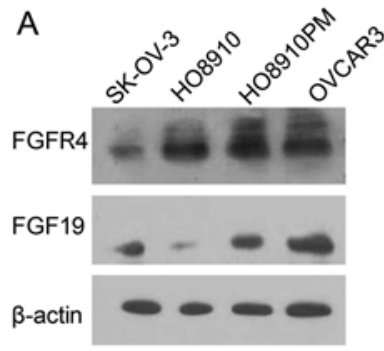

E
B

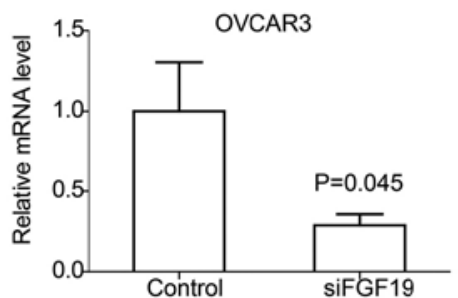

C

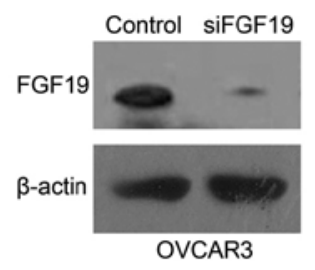

D

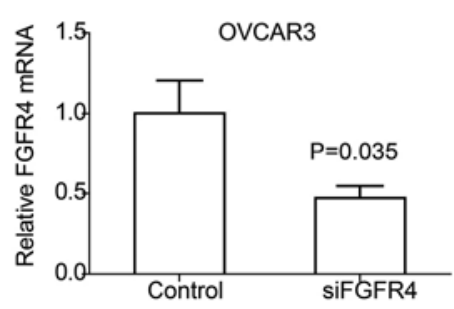

$\mathrm{F}$

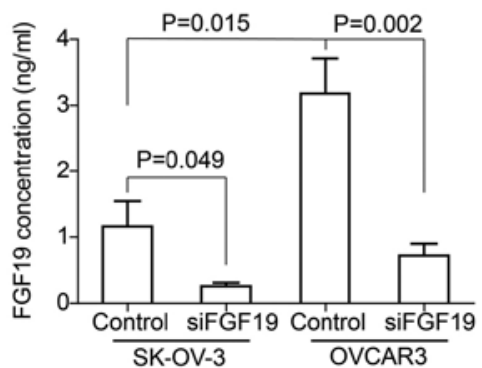

G

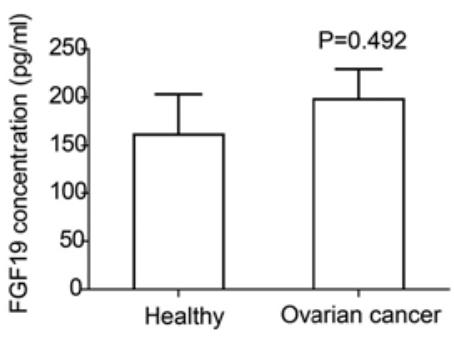

Figure 3. FGF19 and FGFR4 expression in ovarian cancer cells. (A) The expression of FGF19 and FGFR4 was detected in ovarian cancer cell lines SK-OV-3, HO8910, HO8910PM and OVCAR3 by immunoblotting. (B and C) FGF19 knockdown was validated by (B) qRT-PCR and (C) immunoblotting in the OVCAR3 cell line. (D and E) FGFR4 knockdown was validated by (D) qRT-PCR and (E) immunoblotting in the OVCAR3 cell line. (F) FGF19 concentration in the medium of OVCAR3 was significantly higher than the concentration of FGF19 in SK-OV-3 cells. (G) FGF19 concentration in serum was detected by ELISA method in healthy people and patients suffering from ovarian cancer. Statistical data were from three independent experiments and shown as mean \pm SEM.

A

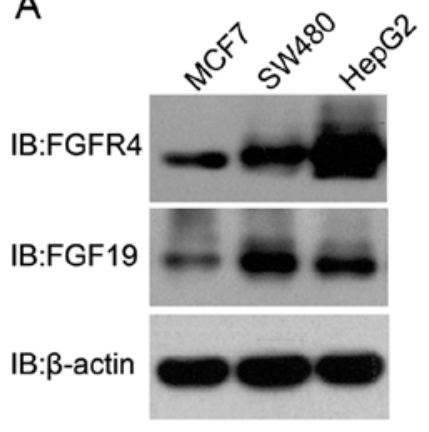

B

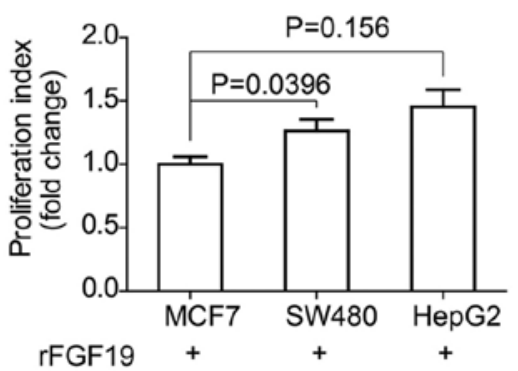

C FGFR4 knockdown

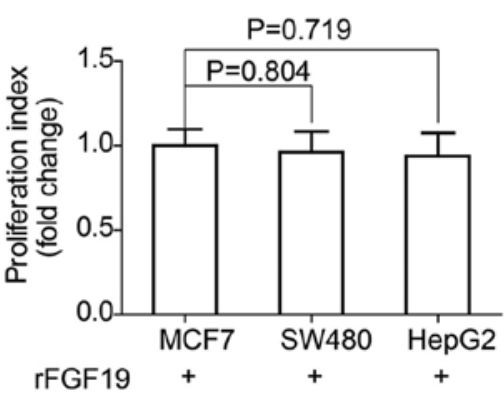

Figure 4. (A) The expression of FGFR4 and FGF19 in hepatocellular carcinoma cell line HepG2, colorectal cancer cell line SW480 and breast cancer cell line MCF7. (B) HepG2, SW480 and MCF7 cells exhibited different proliferative rates under $10 \mathrm{ng} / \mathrm{ml}$ recombinant FGF19 for $48 \mathrm{~h}$. (C) The differential response to $10 \mathrm{ng} / \mathrm{ml}$ recombinant FGF19 stimulation of HepG2, SW480 and MCF7 cells decreased when FGFR4 was knocked down.

poorer prognosis. Thus, we detected the FGF19 concentration in 12 healthy individuals and 12 pre-operational patients with advanced-stage ovarian cancer by ELISA method. However, the FGF19 concentration between the healthy individuals and the ovarian cancer patients had no significant difference $(\mathrm{P}=0.492)$ (Fig. 3G).

Additionally, we detected the expression and function of FGF19 and FGFR4 in control cell lines MCF7, SW480 and HepG2 to determine the exclusive effect of FGF19 on FGFR4. HepG2 had the highest FGFR4 expression and SW480 exhibited the most abundant FGF19 expression (Fig. 4A). Under $10 \mathrm{ng} / \mathrm{ml}$ recombinant FGF19 stimulation for $48 \mathrm{~h}$, these three cell lines exhibited different proliferative rates (HepG2 had the highest and MCF7 had the lowest rate) (Fig. 4B). Moreover, this variation to FGF19 stimulation faded away after FGFR4 was knocked down in these cell lines (Fig. 4C), indicating that FGF19 accelerated the proliferation dependent on FGFR4 existence.

FGF19 promotes ovarian cancer progression in a paracrine manner. To explore the role of FGF19 in ovarian cancer progression, we further examined the effect of exogenous FGF19 on the AKT-MAPK signaling pathway in OVCAR3 cells. Proteins in the AKT-MAPK signaling pathway were detected after $10 \mathrm{ng} / \mathrm{ml} \mathrm{rFGF19} \mathrm{stimulation} \mathrm{for}$ 0-30 min (Fig. 5A). The phosphorylation level of FGFR4, AKT, Erk and p38 increased notably along with the FGF19 stimulation, indicating that FGF19 promoted FGFR4 phosphorylation and activated the AKT-MAPK signaling pathway. Although considered as a specific ligand of FGFR4, FGF19 was also proven to interact with FGFR1, FGFR2 and FGFR3 in the presence of $\beta$-klotho (15-17). To evaluate whether 
A

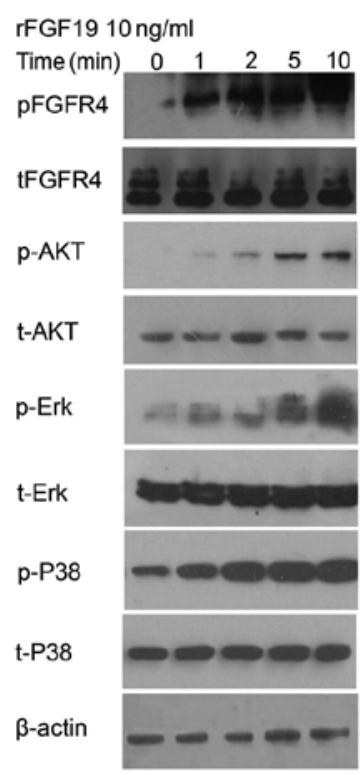

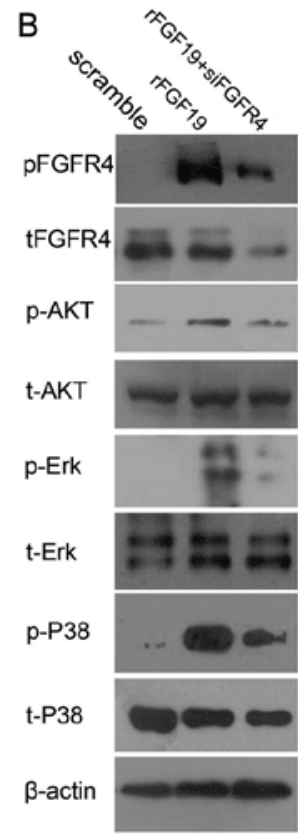

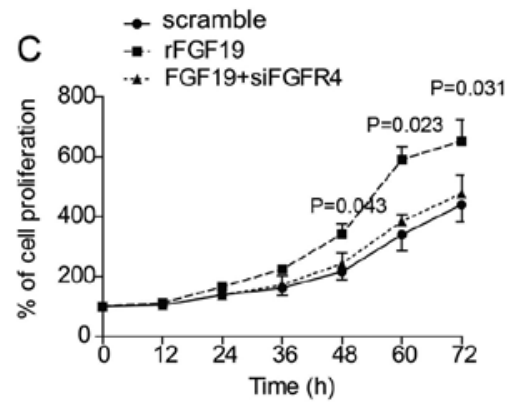

D
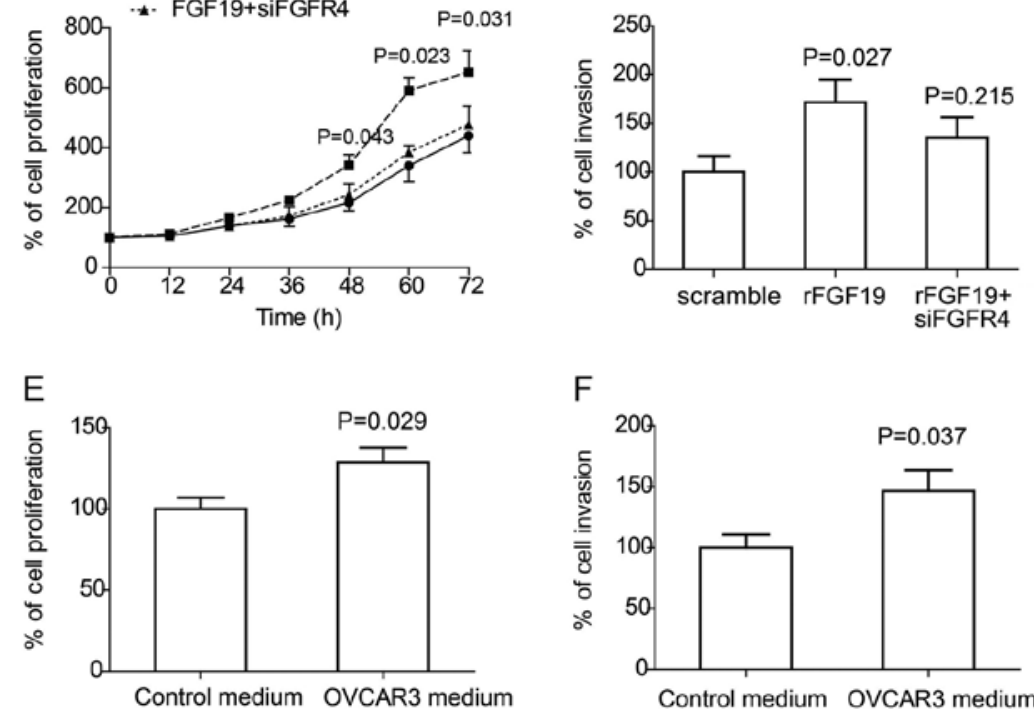

Figure 5. FGF19 promotes ovarian cancer progression by stimulating FGFR4. (A) rFGF19 stimulates FGFR4 and the downstream AKT-Erk/p38 signaling pathway by increasing the phosphorylation in OVCAR3 cells. (B) FGFR4 was required in the FGF19-induced phosphorylation of the AKT-Erk/p38 pathway in OVCAR3 cells. rFGF19 is the group with rFGF19 stimulation after transfection with scrambled siRNA. (C) Recombinant FGF19 promoted proliferation of OVCAR3 cells, while FGFR4 knockdown impaired FGF19-induced proliferation in OVCAR3 cells. rFGF19 is the group with rFGF19 stimulation after transfection with scrambled siRNA. (D) FGFR4 was required in recombinant FGF19-induced invasion of OVCAR3 cells. rFGF19 is the group with rFGF19 stimulation after transfection with scrambled siRNA. (E and F) Conditioned medium from OVCAR3 cells promotes the (E) proliferation and (F) invasion of SK-OV-3 cells.

FGFR4 was essential in the FGF19 activation process, FGFR4 was knocked down in OVCAR3 cells and the AKT-MAPK signaling pathway with recombinant FGF19 stimulation was subsequently detected (Fig. 5B). After FGFR4 knockdown, the phosphorylation levels of AKT, Erk and p38 were significantly reduced due to the decrease in FGFR4 phosphorylation, which demonstrated that FGFR4 was essentially required in the FGF19-induced AKT-MAPK activation.

To explore FGF19-FGFR4 signaling in tumor progression, $10 \mathrm{ng} / \mathrm{ml}$ FGF19 was used to stimulate OVCAR3 cells for 0-72 $\mathrm{h}$ after FGFR4 knockdown, and MTT assay was performed to evaluate cell proliferation (Fig. 5C). Cells with FGF19 stimulation had an obviously higher proliferation rate than the control group, and FGFR4 knockdown reversed this FGF19-induced acceleration of proliferation, indicating that FGF19 promoted ovarian cancer cell proliferation by activating FGFR4. To estimate FGF19 influence on invasion, OVCAR3 cells were cultured in $10 \mathrm{ng} / \mathrm{ml} \mathrm{rFGF19}$ for $24 \mathrm{~h}$, at which time the proliferation did not differ, and then the Matrigel assay was carried out to detect cell invasion (Fig. 5D). In our experiment, FGF19 markedly promoted cell invasion and FGFR4 knockdown impaired this tendency, which confirmed that FGFR4 was also required in the FGF19-induced invasion. Based on our findings that FGF19 was secreted by ovarian cancer cells, we suspected that the FGF19-FGFR4 signaling pathway may be stimulated ectopically by an autocrine or paracrine pathway. Thus, we used the conditioned medium of OVCAR3, the cell line with high FGF19 expression, to stimulate SK-OV-3 cells to confirm our hypothesis. In our experiments, the conditioned medium of OVCAR3 cells notably accelerated the proliferation of SK-OV-3 cells at $72 \mathrm{~h}$ and the invasion of SK-OV-3 at $24 \mathrm{~h}$ (Fig. 5E and F), demonstrating that the FGF19 concentration in the ovarian cancer microenvironment was high enough to activate FGFR4 and downstream signaling pathways.

\section{Discussion}

In our experiments, we detected the expression of FGF19, the FGFR4 specific ligand, in advanced-stage ovarian cancer tissues, and systemically evaluated the correlation between FGF19 and clinicopathological factors and the overall survival for the first time according to our knowledge. Moreover, we performed a series of experiments in vitro to estimate the role of the FGF19-FGFR4 signaling pathway in ovarian cancer progression.Consequently, we found thatFGF19 expression was significantly associated with lymphatic metastasis $(\mathrm{P}=0.033)$. FGF19 and FGFR4 expression could predict poorer prognosis ( $\mathrm{P}=0.033$ and 0.018 , respectively), and FGF19-FGFR4 double high expression was a more accurate and sensitive prognostic factor in ovarian cancer $(\mathrm{P}<0.001)$. With experiments in vitro, we found that FGF19 existed in ovarian cancer cells and was secreted out. In addition, FGF19 stimulated the AKT-MAPK signaling pathway via activating FGFR4, by which FGF19 accelerated proliferation and invasion. Moreover, we proved that the FGF19 concentration in cell culture medium was high enough to promote cell proliferation and invasion, indicating that the FGF19-FGFR4 signaling pathway may be activated ectopically by an autocrine or paracrine pathway. 
FGFR4 overexpression has been previously proven to be associated with progression and prognosis in many types of cancers, including gastric cancer, hepatocellular carcinoma, cholangiocarcinoma, colorectal and oropharynx cancer (18-22). As the known specific ligand to FGFR4, FGF19 was also demonstrated to be involved in progression of cancers such as hepatocellular carcinoma, prostate cancer $(12,18,22,23)$ and colon cancer. The amplification of the FGFR4 gene was discovered in gynecological cancers by Jaakkola et al in 1993 (24). A previous study pointed out that FGFR4 could predict prognosis and may be a potential therapeutic target in high-advanced ovarian cancer (10). However, the FGFR4 ligand was not detected and the underlying molecular mechanism was not well elucidated, which prompted us to explore the correlation between FGF19, FGFR4, progression and prognosis in ovarian cancer. Moreover, FGFR4 is widely expressed in many types of tumors and we demonstrated that not only ovarian cancer cell lines but also other cell lines exhibited a different response to FGF19-induced proliferation, which required the participation of FGFR4. Thus, FGF19-induced proliferation may be a general phenomenon regarding all cell lines with abundant FGFR4, indicating that the FGF19-FGFR4 signaling pathway may be a potential molecular target for ovarian cancer as well as other tumors with FGFR4 overexpression.

Among the human FGF factors, FGF19 is distinguished by its function as a hormone, regulating bile acid synthesis, with effects on glucose and lipid metabolism $(25,26)$. Interestingly, ovarian cancer risk is believed to be positively correlated with glucose and lipid metabolism disorders such as obesity and diabetes (27,28). Recent evidence indicates that obesity, diabetes and ovarian cancer are more intricately related $(29,30)$. Previous studies reported that diet and diabetes are associated with mortality and the prognosis of ovarian cancer $(28,31)$. Based on our new findings, we boldly hypothesize that FGF19 ectopic expression and function may be one explanation why obesity and diabetes are related to high-risk or even a poorer prognosis of ovarian cancer. In our study, the FGF19 concentration of blood samples was detected but no significant difference between the patients and healthy individuals was observed. We believe that more cases should be enrolled and the cohort should be further divided into subgroups according to suspicious parameters such as diabetes and BMI to detect the role of FGF19 in the correlation between ovarian cancer and lipid metabolism.

The FGFR family has five members and can interact with several FGFs with different affinity, which may trigger different downstream signaling pathways and regulate different cellular biological processes $(32,33)$. Moreover, the FGFR family is also proven to undergo crosstalk with other receptors such as the epidermal growth factor receptor family, which may increase the complexity of the FGFR signaling network exponentially (34). Even in ovarian cancer, the FGF19-FGFR4 signaling pathway is associated with obvious conundrums to solve, such as the difference in FGF19 secretion between biological and pathological patterns, and how FGF19 interacts with FGFR4 and which downstream pathways are dominantly triggered. These questions require further fundamental experiments, especially using animal models. Moreover, inhibitors mimicking the FGF19 structure are potential molecular drugs targeting FGFR4 in ovarian cancer based on our finding that
FGF19 is also a prognostic marker. We hope our findings stimulate more research on the function of FGF19 in ovarian cancer, which may further help identify effective therapeutic drugs and increase the survival of patients with ovarian cancer.

In conclusion, we demonstrated that both FGF19 and FGFR4 overexpression predict an unfavorable prognosis in ovarian cancer, while FGF19-FGFR4 double high expression is a better and more sensitive prognostic biomarker. Moreover, either recombinant FGF19 or FGF19 secreted by ovarian cancer cells promotes proliferation and invasion by FGFR4 activation and the subsequent AKT-MAPK signaling pathway. This indicates that FGF19-FGFR4 signaling may be auto-activated in a paracrine or autocrine manner and that FGF19 could be a potential drug target for ovarian cancer.

\section{References}

1. Jemal A, Thomas A, Murray T and Thun M: Cancer statistics, 2002. CA Cancer J Clin 52: 23-47, 2002.

2. Colombo PE, Fabbro M, Theillet C, Bibeau F, Rouanet P and Ray-Coquard I: Sensitivity and resistance to treatment in the primary management of epithelial ovarian cancer. Crit Rev Oncol Hematol 89: 207-216, 2014.

3. Siegel R, Naishadham D and Jemal A: Cancer statistics, 2012. CA Cancer J Clin 62: 10-29, 2012.

4. Seltzer V; NIH Consensus Development Panel on Ovarian Cancer: NIH consensus conference. Ovarian cancer. Screening, treatment, and follow-up. JAMA 273: 491-497, 1995.

5. Kang KW, Lee MJ, Song JA, Jeong JY, Kim YK, Lee C, Kim TH, Kwak KB, Kim OJ and An HJ: Overexpression of goosecoid homeobox is associated with chemoresistance and poor prognosis in ovarian carcinoma. Oncol Rep 32: 189-198, 2014.

6. McGuire WP, Hoskins WJ, Brady MF, Kucera PR, Partridge EE, Look KY, Clarke-Pearson DL and Davidson M: Cyclophosphamide and cisplatin compared with paclitaxel and cisplatin in patients with stage III and stage IV ovarian cancer. $\mathrm{N}$ Engl J Med 334: 1-6, 1996.

7. Turner N and Grose R: Fibroblast growth factor signalling: From development to cancer. Nat Rev Cancer 10: 116-129, 2010.

8. Eswarakumar VP, Lax I and Schlessinger J: Cellular signaling by fibroblast growth factor receptors. Cytokine Growth Factor Rev 16: 139-149, 2005.

9. Wiedemann M and Trueb B: Characterization of a novel protein (FGFRL1) from human cartilage related to FGF receptors. Genomics 69: 275-279, 2000.

10. Zaid TM, Yeung TL, Thompson MS, Leung CS, Harding T, Co NN, Schmandt RS, Kwan SY, Rodriguez-Aguay C, Lopez-Berestein G, et al: Identification of FGFR4 as a potential therapeutic target for advanced-stage, high-grade serous ovarian cancer. Clin Cancer Res 19: 809-820, 2013.

11. Wang D, Zhu W, Li J, An C and Wang Z: Serum concentrations of fibroblast growth factors 19 and 21 in women with gestational diabetes mellitus: Association with insulin resistance, adiponectin, and polycystic ovary syndrome history. PLoS One 8: e81190, 2013 .

12. Feng S, Dakhova O, Creighton CJ and Ittmann M: Endocrine fibroblast growth factor FGF19 promotes prostate cancer progression. Cancer Res 73: 2551-2562, 2013.

13. Song KH, Li T, Owsley E, Strom S and Chiang JY: Bile acids activate fibroblast growth factor 19 signaling in human hepatocy tes to inhibit cholesterol 7alpha-hydroxylase gene expression. Hepatology 49: 297-305, 2009.

14. Stejskal D, Karpísek M, Hanulová Z and Stejskal P: Fibroblast growth factor-19: Development, analytical characterization and clinical evaluation of a new ELISA test. Scand J Clin Lab Invest 68: 501-507, 2008.

15. Xie MH, Holcomb I, Deuel B, Dowd P, Huang A, Vagts A, Foster J, Liang J, Brush J, Gu Q, et al: FGF-19, a novel fibroblast growth factor with unique specificity for FGFR4. Cytokine 11: 729-735, 1999.

16. Zhang X, Ibrahimi OA, Olsen SK, Umemori H, Mohammadi M and Ornitz DM: Receptor specificity of the fibroblast growth factor family. The complete mammalian FGF family. J Biol Chem 281: 15694-15700, 2006. 
17. Kurosu H, Choi M, Ogawa Y, Dickson AS, Goetz R, Eliseenkova AV, Mohammadi M, Rosenblatt KP, Kliewer SA and Kuro-o M: Tissue-specific expression of betaKlotho and fibroblast growth factor (FGF) receptor isoforms determines metabolic activity of FGF19 and FGF21. J Biol Chem 282: 26687-26695, 2007

18. Miura S, Mitsuhashi N, Shimizu H, Kimura F, Yoshidome H, Otsuka M, Kato A, Shida T, Okamura D and Miyazaki M: Fibroblast growth factor 19 expression correlates with tumor progression and poorer prognosis of hepatocellular carcinoma. BMC Cancer 12: 56, 2012.

19. Ye YW, Zhou Y, Yuan L, Wang CM, Du CY,Zhou XY,Zheng BQ, Cao X, Sun MH, Fu H, et al: Fibroblast growth factor receptor 4 regulates proliferation and antiapoptosis during gastric cancer progression. Cancer 117: 5304-5313, 2011.

20. Xu YF, Yang XQ, Lu XF, Guo S, Liu Y, Iqbal M, Ning SL, Yang H, Suo N and Chen YX: Fibroblast growth factor receptor 4 promotes progression and correlates to poor prognosis in cholangiocarcinoma. Biochem Biophys Res Commun 446: 54-60, 2014.

21. Dutra RL, de Carvalho MB, Dos Santos M, Mercante AM, Gazito D, de Cicco R, Group G, Tajara EH, Louro ID and da Silva AM: FGFR4 profile as a prognostic marker in squamous cell carcinoma of the mouth and oropharynx. PLoS One 7: e50747, 2012

22. Li CS, Zhang SX, Liu HJ, Shi YL, Li LP, Guo XB and Zhang ZH: Fibroblast growth factor receptor 4 as a potential prognostic and therapeutic marker in colorectal cancer. Biomarkers 19: 81-85, 2014.

23. Sawey ET, Chanrion M, Cai C, Wu G, Zhang J, Zender L, Zhao A, Busuttil RW, Yee H, Stein L, et al: Identification of a therapeutic strategy targeting amplified FGF19 in liver cancer by oncogenomic screening. Cancer Cell 19: 347-358, 2011.

24. Jaakkola S, Salmikangas P, Nylund S, Partanen J, Armstrong E, Pyrhönen S, Lehtovirta P and Nevanlinna H: Amplification of fofr4 gene in human breast and gynecological cancers. Int J Cancer 54: 378-382, 1993.

25. Jones SA: Physiology of FGF15/19. Adv Exp Med Biol 728: $171-182,2012$.
26. Potthoff MJ, Kliewer SA and Mangelsdorf DJ: Endocrine fibroblast growth factors 15/19 and 21: From feast to famine. Genes Dev 26: 312-324, 2012

27. Doll KM, Kalinowski AK, Snavely AC, Irwin DE, Bensen JT, Bae-Jump VL, Kim KH, Van Le L, Clarke-Pearson DL and Gehrig PA: Obesity is associated with worse quality of life in women with gynecologic malignancies: An opportunity to improve patient-centered outcomes. Cancer 121: 395-402, 2014.

28. Shah MM, Erickson BK, Matin T, McGwin G Jr, Martin JY, Daily LB, Pasko D, Haygood CW, Fauci JM and Leath CA III: Diabetes mellitus and ovarian cancer: More complex than just increasing risk. Gynecol Oncol 135: 273-277, 2014.

29. Thomson CA, E Crane T, Wertheim BC, Neuhouser ML, Li W, Snetselaar LG, Basen-Engquist KM, Zhou Y and Irwin ML: Diet quality and survival after ovarian cancer: Results from the Women's Health Initiative. J Natl Cancer Inst 106: 106, 2014.

30. Olsen CM, Nagle CM, Whiteman DC, Ness R, Pearce CL, Pike MC, Rossing MA, Terry KL, Wu AH, Risch HA, et al; Australian Cancer Study (Ovarian Cancer); Australian Ovarian Cancer Study Group; Ovarian Cancer Association Consortium: Obesity and risk of ovarian cancer subtypes: Evidence from the Ovarian Cancer Association Consortium. Endocr Relat Cancer 20: 251-262, 2013.

31. Bae HS, Kim HJ, Hong JH, Lee JK, Lee NW and Song JY: Obesity and epithelial ovarian cancer survival: A systematic review and meta-analysis. J Ovarian Res 7: 41, 2014.

32. Katoh $\mathrm{M}$ and Nakagama H: FGF receptors: Cancer biology and therapeutics. Med Res Rev 34: 280-300, 2014.

33. Kotsopoulos J, Moody JR, Fan I, Rosen B, Risch HA, McLaughlin JR, Sun P and Narod SA: Height, weight, BMI and ovarian cancer survival. Gynecol Oncol 127: 83-87, 2012.

34. Ding W, Shi W, Bellusci S, Groffen J, Heisterkamp N, Minoo P and Warburton D: Sprouty2 downregulation plays a pivotal role in mediating crosstalk between TGF-betal signaling and EGF as well as FGF receptor tyrosine kinase-ERK pathways in mesenchymal cells. J Cell Physiol 212: 796-806, 2007. 